\title{
Long intergenic non-protein coding RNA 00460 predicts a poor prognosis and promotes tumorigenesis of human osteosarcoma
}

\author{
JIAN-JUN JIANG, FENG-CHAO WANG and LI-PING HAN \\ Department of Orthopedics, Zaozhuang Municipal Hospital, Zaozhuang, Shandong 277100, P.R. China
}

Received May 13, 2019; Accepted October 31, 2019

DOI: $10.3892 / \mathrm{mmr} .2019 .10858$

\begin{abstract}
Osteosarcoma (OS) is the most common type of primary malignant bone tumor, which has a high incidence rate in children and adolescents. This research aims to reveal the role of long intergenic non-protein coding RNA 00460 (LINC00460) in OS by the loss-of-function experiment. LINC00460 is involved in the development of multiple types of tumor, but the role of LINC00460 in OS is unclear. To discover more effective molecular targets for the treatment of OS, the association between LINC00460 and OS prognosis was analyzed using the Gene Expression Profiling Interactive Analysis database. Additionally, small interfering RNA was used to knockdown LINC00460 gene expression in vitro to verify its biological effects on the viability, invasive and migratory potential of OS cells. LINC00460 knockdown significantly reduced the viability of OS cells and initiated cell cycle arrest within the G0/G1 phase through the decreased expression of cyclin D1 and CDK4/CDK6. In addition, LINC00460 knockdown promoted apoptosis of OS cells, and inhibited the migratory and invasive abilities of OS cells through the inhibition of the epithelial-mesenchymal transition pathway. In conclusion, the present study reported that LINC00460 may predict OS prognosis, and may serve an important role in mediating the viability, invasive and migratory potential of OS cells. Based on these findings, LINC00460 demonstrated promising potential as a future therapeutic target for OS treatment.
\end{abstract}

\section{Introduction}

Osteosarcoma (OS) is the most common type of primary malignant bone tumor, and demonstrates a high degree of malignancy, invasion, metastasis and recurrence that significantly affects patient prognosis; OS accounts for $\sim 5 \%$ of cases

Correspondence to: Dr Li-Ping Han, Department of Orthopedics, Zaozhuang Municipal Hospital, 41 Longtou Road, Zaozhuang, Shandong 277100, P.R. China

E-mail: hanliping007@outlook.com

Key words: long intergenic non-protein coding RNA 00460, osteosarcoma, cell cycle arrest, apoptosis, epithelial-mesenchymal transition of childhood cancer (1-3). Currently, clinical treatment of OS consists of a combination of chemotherapy, radiation therapy and surgery, which has demonstrated limited improvement in OS survival during the last decade. Unfortunately, $30 \%$ of patients with metastatic or recurrent disease will not survive for $>5$ years (3-5). Thus, there remains an urgent requirement to discover improved therapeutic targets that have the potential to be developed into alternative treatment strategies that could improve the survival rate of patients with OS.

Long non-coding RNAs (lncRNAs) are transcripts of $\sim 200$ nucleotides and $\sim 10 \mathrm{~kb}$ in length (6-9), which serve numerous biological functions across many life processes, including cell cycle control, development and differentiation $(10,11)$. Recently, there has been increased focus on lncRNAs due to their crucial role in the progression of multiple types of cancer, including OS $(12,13)$. For example, lncRNA H19 is aberrantly expressed and induced by upregulated Hedgehog signaling and Yes-associated protein 1 overexpression, and is responsible for the pathogenesis of osteoblastic OS (14). The oncogenic lncRNA metastasis-associated lung adenocarcinoma transcript 1 has been reported to serve as a competing endogenous RNA of histone deacetylase 4 by decoying microRNA-140-5p to promote OS tumor growth (15). However, it remains relatively unknown whether other lncRNAs have roles in OS.

Among the wide variety of lncRNAs, long intergenic non-protein coding RNA 00460 (LINC00460) is of importance. It has been reported that LINC00460 is involved in the progression of multiple types of solid carcinoma, such as kidney carcinoma, lung adenocarcinoma, head and neck squamous cell carcinoma and epithelial ovarian cancer (16-21). However, to the best of our knowledge, the role of LINC00460 in OS has not yet been reported. Through online data analysis using the Gene Expression Profiling Interactive Analysis (GEPIA) database, which includes RNA sequencing expression data of 9,736 tumors and 8,587 normal samples from The Cancer Genome Atlas and The Genotype-Tissue Expression projects, it was observed in the present study that the overall prognosis of patients with low LINC00460 expression in sarcoma was significantly improved compared with patients with high LINC00460 expression. This implied that LINC00460 may be involved in the progression of OS.

In the present study, a series of experiments were performed to detect the roles of LINC00460 in OS. It was determined that LINC00460 knockdown with small interfering RNA (siRNA) 
inhibited cell viability, and migratory and invasive potential of OS cells, which may be related to its ability to cause cell cycle arrest, apoptosis and reduced epithelial-mesenchymal transition (EMT) of OS cells.

\section{Materials and methods}

Chemicals and reagents. DMEM and RPMI-1640 medium were purchased from HyClone; GE Healthcare Life Sciences, FBS was obtained from Gibco; Thermo Fisher Scientific, Inc. and Lipofectamine ${ }^{\circledR} 2000$ from Invitrogen; Thermo Fisher Scientific, Inc. Matrigel was obtained from BD Biosciences, and siRNA-LINC00460 and the scrambled siRNA were synthesized by Guangzhou Ribobio Co., Ltd. The following primary antibodies: Anti-CDK4 (1:1,000; cat. no. 12790S), anti-CDK6 (1:1,000; cat. no. 3136P), anti-vimentin (1:1,000; cat. no. 3878S), anti-E-cadherin (1:1,000; cat. no. 4065), anti-N-cadherin (1:1,000; cat. no. 4061P), anti-cyclin D1 (1:1,000; cat. no. 2978P), anti-Slug (1:1,000; cat. no. 9585S) and anti-GAPDH (1:5,000; cat. no. 2118L) were purchased from Cell Signaling Technology, Inc. Goat anti-rabbit or goat anti-mouse horseradish peroxidase (HRP)-conjugated secondary antibodies (cat. nos. G1215 and G1216) and ECL reagent were obtained from Wuhan Sanying Biotechnology. The propidium iodide (PI) was purchased from Beyotime Institute of Biotechnology, and the Cell Counting Kit (CCK)-8, trypsin, penicillin, streptomycin sulfate and crystal violet were obtained from Beijing Solarbio Science \& Technology Co., Ltd. Unless otherwise stated, other reagents were obtained from CoWin Biosciences Co., Ltd.

Cell lines and culture. OS cell lines MG-63 (TCHu124) and U2-OS (SCSP-5030) were obtained from the Shanghai Cell Bank of Chinese Academy of Medical Sciences and cultured in DMEM supplemented with $10 \%$, penicillin $(100 \mathrm{U} / \mathrm{ml})$ and streptomycin sulfate $(100 \mu \mathrm{g} / \mathrm{ml})$. All cells were maintained in a humidified incubator at $37^{\circ} \mathrm{C}$ and $5 \% \mathrm{CO}_{2}$. During the logarithmic growth phase, cells were washed three times in PBS and digested with trypsin to obtain single cell suspensions. The cells were subsequently plated out and cultured in 6-well plates for further experimentation.

Cell transfection. OS cells in the logarithmic growth phase were replenished with fresh DMEM $2 \mathrm{~h}$ prior to transfection. The siRNA sequences against LINC00460 or the negative control (NC) were designed and synthesized by Guangzhou RiboBio Co., Ltd. siRNA ( $3 \mu \mathrm{g} / \mathrm{ml}$ ) was transfected into $3 \times 10^{4} \mathrm{OS}$ cells at a cell density of $3 \times 10^{4}$ cells for $6 \mathrm{~h}$ at $37^{\circ} \mathrm{C}$ using Lipofectamine ${ }^{\circledR}$ 2000 transfection reagent, according to the manufacturer's protocol. Subsequently, the medium was replaced and the cells were cultured in DMEM for $48 \mathrm{~h}$ for subsequent experiments. The sequences of the LINC00460 siRNAs were as follows: NC, 5'-UUCUCCGAACGUGUCACGUTT-3'; LINC00460-1, 5'-CCAUCCACUUCA AAGUAUUTT-3'; LINC00460-2, 5'-GCCUCUGAA AUGGUGACAATT-3'; LINC00460-3, 5'-GGGAAAGAAGACGCAUUCUTT-3' and LINC00460-4, 5'-UCACCUUGACUACUGCUAUTT-3'.

Reverse transcription-quantitative PCR (RT-qPCR). Total RNA was extracted from transfected cells using an UltraPure
RNA kit (CoWin Biosciences Co., Ltd.), according to the manufacturer's protocol. Total RNA was reverse transcribed into cDNA using a HiFiScript cDNA Synthesis kit (CoWin Biosciences Co., Ltd.). The reverse transcription reaction conditions were as follows: Incubation at $42^{\circ} \mathrm{C}$ for $50 \mathrm{~min}$, followed by incubation at $85^{\circ} \mathrm{C}$ for $5 \mathrm{~min}$ to terminate the reaction. qPCR was performed to assess the silencing effects of siRNA using the UltraSYBR mixture (CoWin Biosciences Co., Ltd.). The following primer pairs were used for the qPCR: LINC00460 forward, 5'-CAGAAATCCTCCAGCCCTGTT A-3' and reverse, 5'-AAGTGTCTTGGGTCATGAGTCC-3'; and $\beta$-actin forward, 5'-CCCGAGCCGTGTTTCCT-3' and reverse, 5'-GTCCCAGTTGGTGACGATGC-3'. The following thermocycling conditions were used for qPCR: Initial denaturation at $95^{\circ} \mathrm{C}$ for $30 \mathrm{sec} ; 40$ cycles of $95^{\circ} \mathrm{C}$ for $5 \mathrm{sec}, 60^{\circ} \mathrm{C}$ for $30 \mathrm{sec}$ and one cycle of melting curve at $95^{\circ} \mathrm{C}$ for $15 \mathrm{sec}, 60^{\circ} \mathrm{C}$ for $1 \mathrm{~min}, 95^{\circ} \mathrm{C}$ for $15 \mathrm{sec}$ and $50^{\circ} \mathrm{C}$ for $30 \mathrm{sec}$. Expression levels were quantified using the $2^{-\Delta \Delta \mathrm{Cq}}$ method (22) from three independent experiments and normalized to the internal reference gene $\beta$-actin.

Cell viability assay. Cell viability was determined using a CCK-8 assay. After $24 \mathrm{~h}$ of transfection with siRNA, cells were trypsinized and resuspended in DMEM; $100 \mu$ l of the cell suspension was seeded into 96-well plates at a density of $1 \times 10^{3}$ cells/well. Next, $10 \mu$ l CCK-8 reagent was added into each well and cells were incubated in a humidified incubator at $37^{\circ} \mathrm{C}$ and $5 \% \mathrm{CO}_{2}$ for $1.5 \mathrm{~h}$. Optical density (OD) values were determined by measuring the absorbance at a wavelength of $450 \mathrm{~nm}$ using a microplate reader. Cell viability was determined every $24 \mathrm{~h}$.

Colony formation assay. A total of $\sim 800$ MG-63 or U2-OS cells/well were seeded into 6-well culture plates for colony formation assays and incubated in the culture medium for 14 days. Subsequently, cells were fixed with $4 \%$ methanol for $20 \mathrm{~min}$ and stained with $0.1 \%$ crystal violet for $30 \mathrm{~min}$ at $37^{\circ} \mathrm{C}$. The colonies were visualized and quantified by counting stained colonies containing $\geq 50$ cells.

Wound healing assay. Cells $\left(1 \times 10^{4}\right.$ cells/well) were seeded in 6-well plates and cultured until the cells reached $95 \%$ confluence. A sterile 200- $\mu l$ tip was used to generate the wound in the monolayer of cells in each well, which was subsequently washed three times and was cultured with serum-free DMEM for $24 \mathrm{~h}$. Subsequently, the cells were visualized using an Olympus IX71 inverted microscope (Olympus Corporation; magnification, $\mathrm{x} 40)$. The area of the wound surface was measured with ImageJ software, version 1.41 (National Institutes of Health). The width of each wound was measured at five random fields for quantification. All analysis was performed relative to the starting wound width $(0 \mathrm{~h}$ time point).

Transwell invasion and Matrigel assays. Cell invasion analysis was performed using a Matrigel-coated Transwell assay. Matrigel was defrosted from $-20^{\circ} \mathrm{C}$ to $4^{\circ} \mathrm{C}$ in a refrigerator overnight and the experiment was performed on ice at all times, with all consumables pre-cooled. A total volume of $100 \mu 1$ diluted Matrigel in serum free-cold DMEM was added to the upper chamber of the Transwell, which was 
subsequently inserted into 24-well plates. After incubating the Transwell at $37^{\circ} \mathrm{C}$ for $4 \mathrm{~h}$, gelled Matrigel was gently washed with warmed serum-free RPMI-1640 medium. After $24 \mathrm{~h}$ of transfection, the cells were trypsinized and re-suspended in serum-free 1640 medium at a density of $1 \times 10^{5}$ cells $/ \mathrm{ml} ; 100 \mu 1$ of this cell suspension ( $1 \times 10^{4}$ cells) was plated in the upper chamber and $600 \mu 11640$ medium containing 10\% FBS was plated in the lower chamber. After $24 \mathrm{~h}$ of incubation at $37^{\circ} \mathrm{C}$, the non-invasive cells remaining in the upper chamber of the Transwell plate were scraped off with a cotton swab. The invading cells on the lower surface of the chamber were fixed with $4 \%$ formaldehyde for $15 \mathrm{~min}$ at room temperature and subsequently stained with $0.1 \%$ crystal violet for $5 \mathrm{~min}$. After washing with PBS, invasive cells were counted using a light microscope (magnification, x100).

Cellular migration was also detected by a Transwell assay and followed the same protocol as the Matrigel invasion assay, with the exception that Matrigel was not used to coat the Transwell plates.

Flow cytometry. After $24 \mathrm{~h}$ of transfection, cells were removed from the culture medium and incubated in serum-free DMEM for $24 \mathrm{~h}$. Subsequently, $1 \times 10^{6}$ cells were trypsinized with EDTA-free-trypsin and harvested by centrifugation $(984 \mathrm{x} \mathrm{g}$; $20^{\circ} \mathrm{C} ; 5 \mathrm{~min}$ ). Cells were resuspended with ice-cold PBS and harvested by centrifugation (same as previous). Cells were fixed overnight with $70 \%$ ice-cold ethanol and then collected by centrifugation $\left(984 \mathrm{x} \mathrm{g} ; 20^{\circ} \mathrm{C} ; 5 \mathrm{~min}\right)$. Cells were resuspended in $100 \mu \mathrm{l}$ PBS containing $100 \mu \mathrm{g} / \mathrm{ml}$ RNase and incubated for $5 \mathrm{~min}$ at room temperature. Subsequently, the cell suspension was added to $400 \mu \mathrm{l}$ PBS containing $50 \mu \mathrm{g} / \mathrm{ml}$ PI at room temperature for $30 \mathrm{sec}$. Following the addition of PI, cells were immediately subjected to FACS analysis by a BD FACSCalibur ${ }^{\mathrm{TM}}$ flow cytometer (BD Biosciences) and FlowJo software, version 4.5 (Tree Star, Inc.) was used to analyze the percentage of cells in each phase of the cell cycle (G0/G1, S or G2/M).

Detection of apoptosis. A $1 \mathrm{mg} / \mathrm{ml}$ stock solution of Hoechst 33342 dye (Beyotime Institute of Biotechnology) and a $0.5 \mathrm{mg} / \mathrm{ml}$ stock solution of PI (Beyotime Institute of Biotechnology) in $\mathrm{H}_{2} \mathrm{O}$ were filter-sterilized. Cells incubated in 24-well plates were washed twice with PBS, then subsequently stained with $10 \mu \mathrm{g} / \mathrm{ml}$ Hoechst 33342 combined with $5 \mu \mathrm{g} / \mathrm{ml} \mathrm{PI}$ at $37^{\circ} \mathrm{C}$ for $15 \mathrm{~min}$, according to the manufacturer's protocol. After washing twice with PBS, the cells were visualized and counted under a fluorescence microscope (magnification, x100).

Gelatin zymography. The enzymatic activities of matrix metalloproteinase (MMP)-9 were analyzed by gelatin zymography, according to previous studies (23-25). A total of $2.5 \times 10^{6}$ cells were seeded in $6-\mathrm{cm}$ dishes and transfection was performed as previously described. After $24 \mathrm{~h}$, cells were washed twice with serum-free DMEM prior to being cultured in serum-free DMEM for $24 \mathrm{~h}$. The culture supernatant was collected by centrifugation $\left(984 \mathrm{x} \mathrm{g} ; 20^{\circ} \mathrm{C} ; 5 \mathrm{~min}\right)$ and the pellet was discarded. Gelatin solution was prepared by dissolving $500 \mathrm{mg}$ gelatin in $50 \mathrm{ml}$ distilled and deionized $\mathrm{H}_{2} \mathrm{O}$ for $2 \mathrm{~h}$ at room temperature, and was subsequently placed in a $65^{\circ} \mathrm{C}$ water bath for $15 \mathrm{~min}$ to obtain a clear gelatin stock solution with a concentration of $10 \mathrm{mg} / \mathrm{ml}$. A $10 \%$ acrylamide gel was prepared and gelatin stock solution was added to obtain a final gelatin concentration of $0.5 \mathrm{mg} / \mathrm{ml}$. $16 \mu \mathrm{l}$ of supernatant was loaded into each well and were run for $1.5-2 \mathrm{~h}$ until the indicator dye reached the bottom of the gel. The gel was washed four times for $15 \mathrm{~min}$ with zymogram renaturing buffer at room temperature, and then the gel was incubated overnight in incubation buffer at $37^{\circ} \mathrm{C}$. Following incubation, the gel was stained with $0.25 \%$ Coomassie Blue R-250 for $4 \mathrm{~h}$ at room temperature, followed by the application of destaining solution (20\% methanol, $10 \%$ acetic acid, $70 \%$ distilled and deionized $\mathrm{H}_{2} \mathrm{O}$ ) with gentle agitation until areas of enzyme activity appeared as transparent bands against the dark blue background. The mixture was rinsed with $\mathrm{H}_{2} \mathrm{O}$ until excess staining solution was removed. PageRuler Prestained Protein Ladder (Thermo Fisher Scientific, Inc.) was used as a marker. The clear bands were visualized using Image Scanner III (GE Healthcare) and the intensity of each band was measured using ImageQuant TL v2003 software (GE Healthcare).

Western blotting. After $48 \mathrm{~h}$ of transfection, total protein from cells transfected with siRNA-NC or siRNA-LINC00460 was extracted using RIPA lysis buffer containing protease inhibitors at $4^{\circ} \mathrm{C}$. Total protein was quantified using a bicinchoninic acid protein assay and $20 \mu \mathrm{g}$ protein/lane was separated via SDS-PAGE on a $10 \%$ gel. The separated proteins were transferred onto a PVDF membrane and blocked for $1 \mathrm{~h}$ at room temperature with $5 \%$ non-fat milk. The membranes were incubated with the corresponding primary antibodies overnight at $4^{\circ} \mathrm{C}$. Membranes were washed three times with TBS- $0.1 \%$ Tween 20 . Following the primary antibody incubation, membranes were incubated with goat anti-rabbit/mouse HRP-conjugated secondary antibodies $(1: 5,000)$ for $1 \mathrm{~h}$ at room temperature. Protein bands were visualized using a chemiluminescence kit. GAPDH served as the loading control. The protein bands intensity was analyzed using Image $\mathbf{J}$ software, version 1.41 (National Institutes of Health).

Statistical analysis. Statistical analysis of all results was performed using SPSS version 20.0 (IBM Corp.) software. Each experiment was independently conducted $\geq 3$ times and all data are expressed as the mean \pm SD. Significant differences between groups were determined using Student's t-test or one-way ANOVA with Bonferroni correction used as the post hoc test. Kaplan Meier analysis was used for survival analysis, and log-rank test was conducted to determine P-values. The prognostic value of LINC00460 in OS was analyzed using the GEPIA database (26). $\mathrm{P}<0.05$ was considered to indicate a statistically significant difference.

\section{Results}

Prognostic value of LINC00460. The overall and disease-free survival of patients with low LINC00460 expression in OS was significantly improved compared with patients with high LINC00460 expression ( $\mathrm{P}<0.05$; Fig. 1A and B).

Genetic knockdown of LINC00460 with siRNA affects OS cell viability. To investigate the function of LINC00460 on 
A

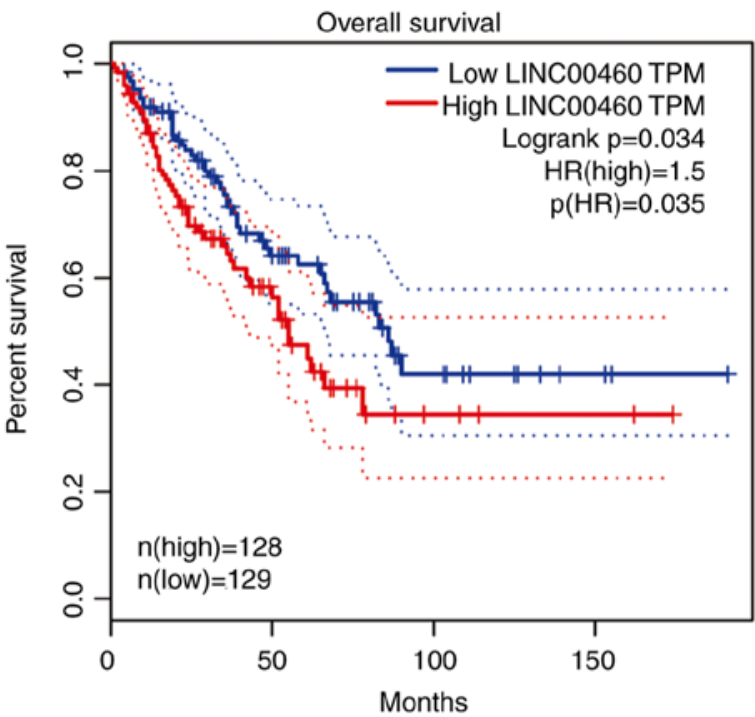

C

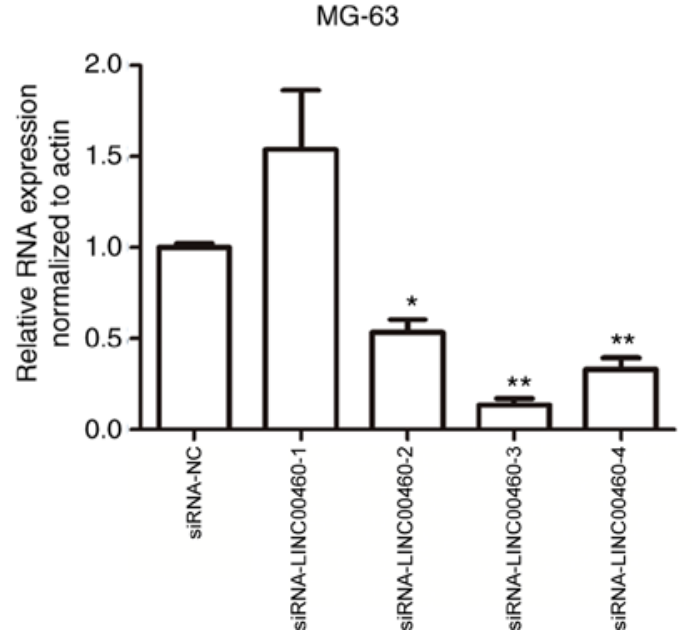

B

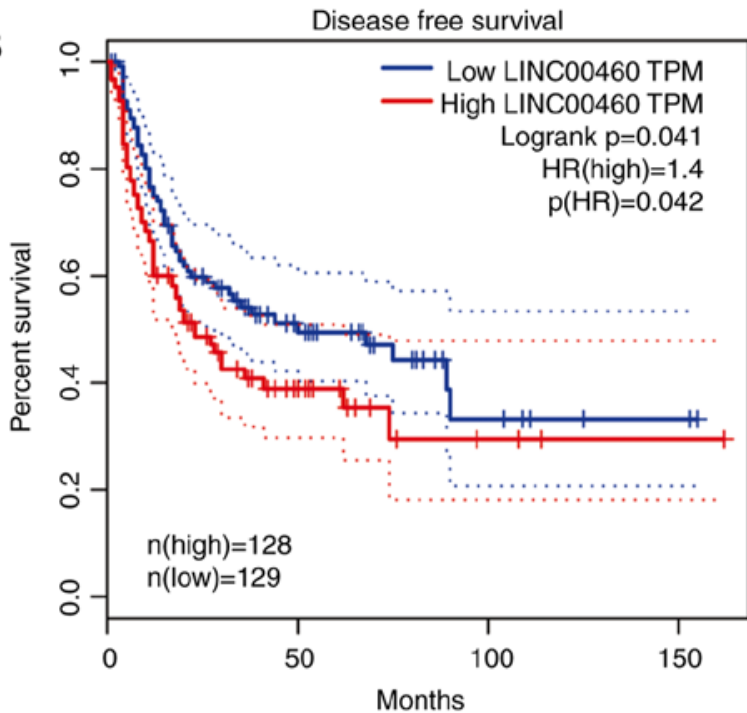

$\mathrm{D}$ U2-OS

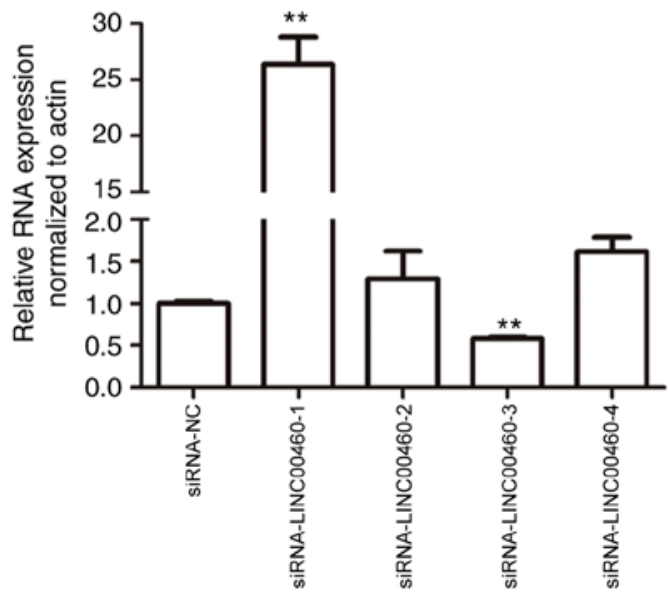

Figure 1. Prognostic value of LINC00460 in OS. The Gene Expression Profiling Interactive Analysis database was used to analyze the prognosis of LINC00460 in OS. Kaplan Meier plots of (A) overall survival and (B) disease-free survival of patients with OS with high or low expression of LINC00460. Reverse transcription-quantitative PCR analysis of LINC00460 expression levels in (C) MG-63 and (D) U2-OS cell lines following transfection with siRNA-NC or siRNA-LINC00460-(1-4). Expression levels were normalized to the internal reference gene $\beta$-actin. " $\mathrm{P}<0.05$ and ${ }^{* *} \mathrm{P}<0.01$ vs. NC. NC, negative control; lncRNA, long non-coding RNA; LINC00460, long intergenic non-protein coding RNA 00460; OS, osteosarcoma; siRNA, small interfering RNA.

OS progression, siRNA was used to knockdown gene expression. Four siRNA fragments, siRNA-LINC00460-(1-4), were designed and synthesized to silence LINC00460 in MG-63 and U2-OS cell lines. siRNA-LINC00460-3 demonstrated the highest siRNA transfection efficiency in MG-63 and U2-OS cell lines, providing a knockdown efficiency of $>36.6$ and $47.1 \%$ compared with $\mathrm{NC}$, respectively $(\mathrm{P}<0.01$; Fig. $1 \mathrm{C}$ and $\mathrm{D})$. Therefore, siRNA-LINC00460-3 was selected as the siRNA fragment used to target LINC00460 for subsequent experiments. To evaluate the effect of LINC00460 on cell viability, CCK-8 and colony formation assays were conducted to detect the effect of LINC00460 knockdown on the viability of MG-63 and U2-OS cell lines. MG-63 and U2-OS cell lines transfected with siRNA-LINC00460-3 demonstrated significantly reduced cell viability by $48-72 \mathrm{~h}$ compared with the $\mathrm{NC}(\mathrm{P}<0.05$; Fig. 2A). Similarly, the colony formation assay reported a significant decrease in the number and size of colonies formed in both cell lines following LINC00460 gene silencing compared with the $\mathrm{NC}$ group $(\mathrm{P}<0.01 ;$ Fig. 2B). These results suggested that the genetic knockdown of LINC00460 significantly affected OS cell viability and growth.

Genetic knockdown of LINC00460 induces apoptosis in OS cells. To further investigate how LINC00460 knockdown affects OS cell proliferation, the effect of LINC00460 on apoptosis was evaluated using Hoechst/PI staining. siRNA-LINC00460transfected OS cells exhibited significant increases in apoptosis compared with the siRNA-NC group (MG-63, 52.93 $\pm 8.25 \%$ vs. $2.61 \pm 0.20 \%$; $\mathrm{U} 2-\mathrm{OS}, 12.33 \pm 2.26 \%$ vs. $2.16 \pm 0.11 \%$; $\mathrm{P}<0.01$; Fig. 2C). These data demonstrated that the genetic knockdown of LINC00460 increased apoptosis of OS cells.

Genetic knockdown of LINC00460 induces the cell cycle arrest of OS cells. To determine which phase of the cell cycle was affected by LINC00460 knockdown, flow cytometric analysis was performed to estimate the distribution of cells at different stages of the cell cycle. The results indicated that siRNA-LINC00460 transfection induced a significant 
A

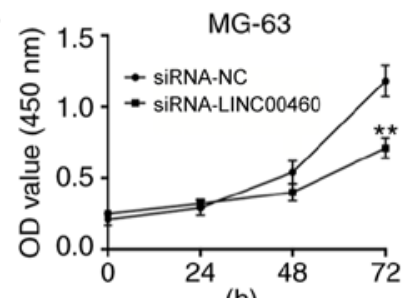

(h)

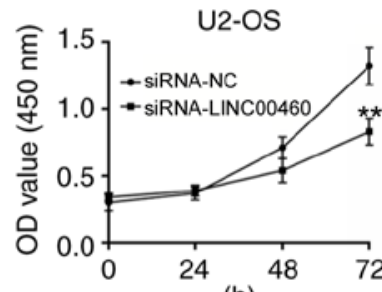

(h)
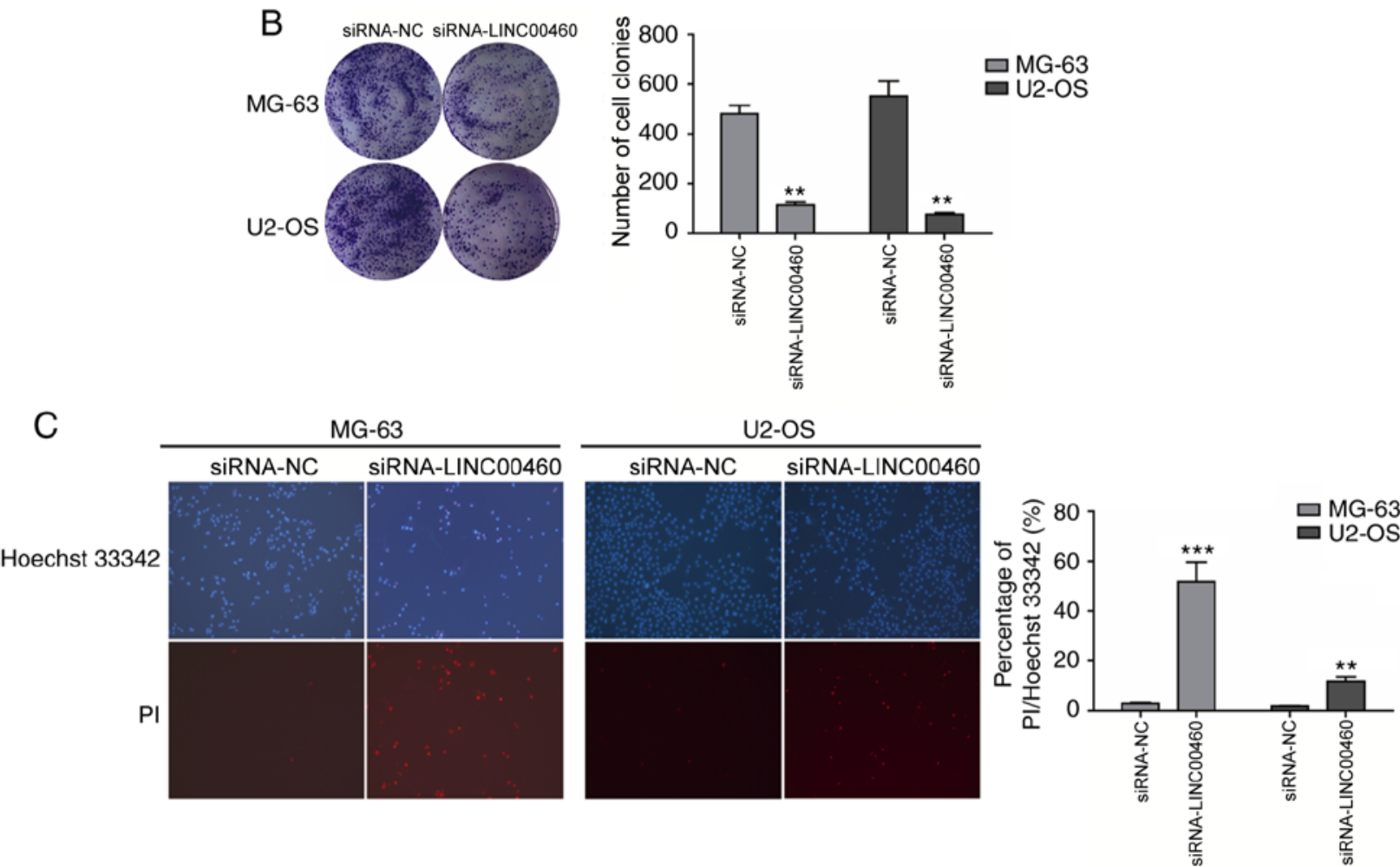

Figure 2. Genetic knockdown of LINC00460 with siRNA inhibits OS cell proliferation and induces apoptosis. (A) CCK-8 assays were performed to determine the cell viability of MG-63 and U2-OS cells following transfection with siRNA-LINC00460 or their respective NC for 24 h. (B) Colony forming assays were conducted to examine the proliferative potential of OS cell lines, MG-63 and U2-OS, following transfection with siRNA-LINC00460 or their respective NC. The quantitative analysis of MG-63 cells and U2-OS cells are provided. (C) Apoptosis and cell death were detected in MG-63 and U2-OS cell lines following transfection with siRNA-LINC00460 or its NC using Hoechst 33342 and PI staining. Representative fluorescent micrographs (magnification, x100) and the quantitative analysis of apoptosis in MG-63 cells and U2-OS cells are displayed. Values are presented as the mean \pm SD of three independent experiments. ${ }^{* * *} \mathrm{P}<0.01$ and ${ }^{* * *} \mathrm{P}<0.001$ vs. NC. NC, negative control; LINC00460, long intergenic non-protein coding RNA 00460; OS, osteosarcoma; siRNA, small interfering RNA; PI, propidium iodide; OD, optical density.

accumulation of cells in the G0/G1 phase of the cell cycle in both MG-63 and U2-OS cell lines compared with the siRNA-NC group (MG-63, 62.1 vs. 32.6\%, respectively; U2-OS, 59.2 vs. $38.1 \%$, respectively; $\mathrm{P}<0.05$; Fig. $3 \mathrm{~A}$ and $\mathrm{B}$ ). This indicated that siRNA-LINC00460 arrested MG-63 and $\mathrm{U} 2-\mathrm{OS}$ cells in the $\mathrm{G} 0 / \mathrm{G} 1$ phase. In addition, the percentage of cells in the G2/M phase significantly decreased in both cell lines compared with the siRNA-NC group (MG-63, $28.4 \pm 3.21 \%$ vs. $10.6 \pm 2.20 \%$, respectively; U2-OS, $31.9 \pm 4.37 \%$ vs. $16.6 \pm 2.78 \%$, respectively; $\mathrm{P}<0.05$; Fig. $3 \mathrm{~A}$ and $\mathrm{B})$. There were no significant changes observed in the $\mathrm{S}$ phase of either cell line. Based on these data, it was hypothesized that siRNA-LINC00460 induced G0/G1 phase arrest and triggered cell apoptosis to reduce cell viability in OS.

Downregulated expression of cyclin D1 and CDK4/CDK6 may induce cell cycle arrest of OS cells in the G0/G1 phase following transfection with siRNA-LINC00460. To further validate the mechanism underlying the G0/G1 phase cell cycle arrest following siRNA-LINC00460 transfection in OS cells, western blot analysis was performed to examine the changes in the protein expression levels of cyclin D1, CDK4 and CDK6 in MG-63 and U2-OS cell lines (Fig. 3C). Cyclin D1, CDK4 and CDK6 expression levels were significantly decreased following the genetic knockdown of LINC00460 with siRNA in both cell lines compared with the siRNA-NC group ( $\mathrm{P}<0.05$; Fig. $3 \mathrm{C})$. These data demonstrated that OS cells were arrested in the G0/G1 phase following transfection with siRNA-LINC00460, which may be caused by the downregulated expression of cyclin D1 and CDK4/CDK6.

Genetic knockdown of LINC00460 inhibits the migratory and invasive abilities of OS cells. Following siRNA-LINC00460 transfection, MG-63 and U2-OS cells demonstrated a significantly reduced migratory ability $(0.22 \pm 0.05$ and $0.28 \pm 0.03$, respectively) compared with the siRNA-NC groups $(0.62 \pm 0.03$ 

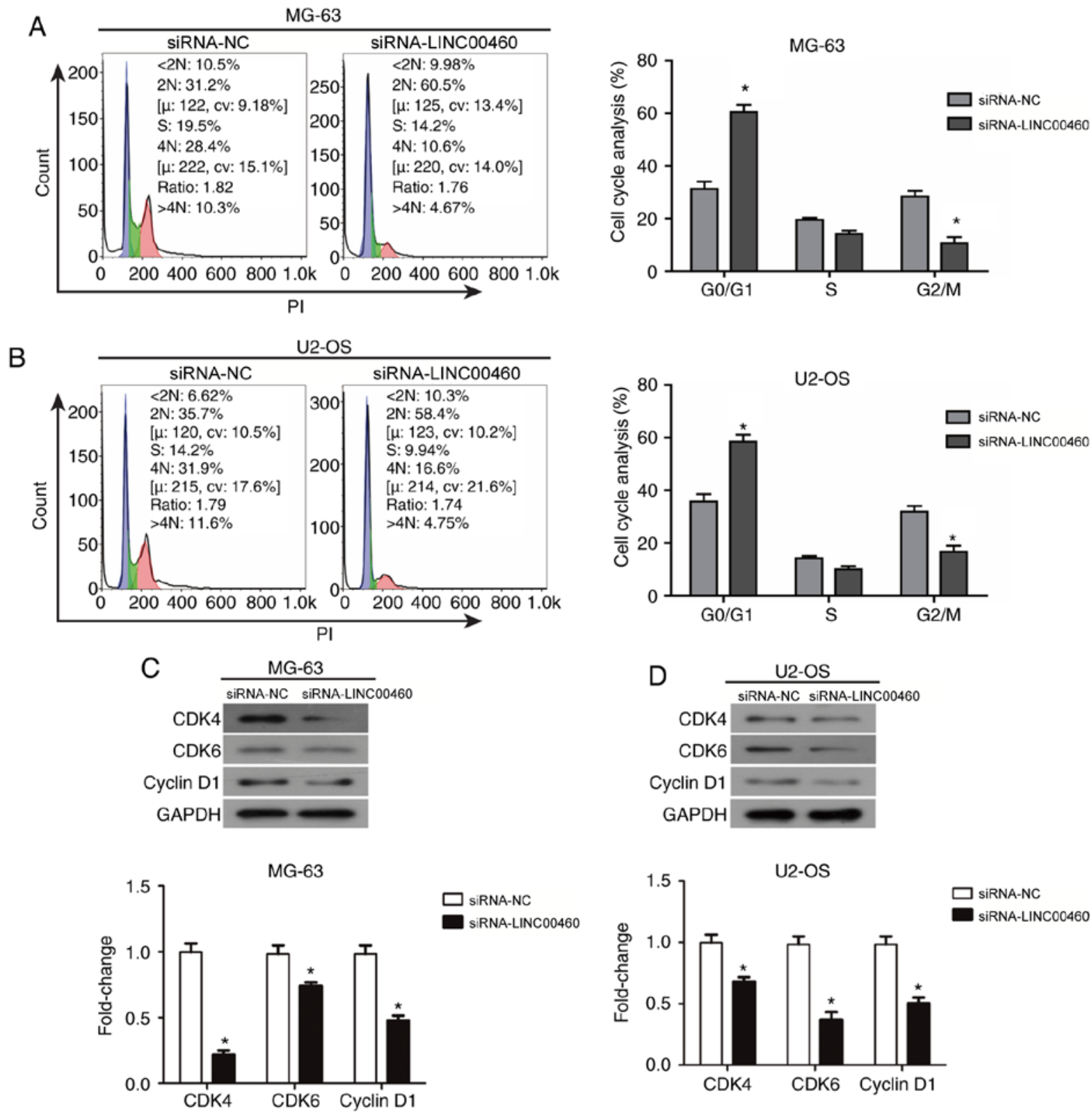

Figure 3. Genetic knockdown of LINC00460 induces cell cycle arrest in osteosarcoma cells. (A and B) Flow cytometric analysis was performed to determine the number of (A) MG-63 and (B) U2-OS cells in each stage of the cell cycle following transfection with siRNA-NC or siRNA-LINC00460. (C and D) Western blotting and semi-quantitative analysis was performed to examine the expression levels of cyclin D1, CDK4 and CDK6 in (C) MG-63 and (D) U2-OS cell lines following transfection with siRNA-NC or siRNA-LINC00460. GAPDH was used as the loading control. All data are presented as the mean \pm SD of three independent experiments. "P<0.05 vs. NC. NC, negative control; LINC00460, long intergenic non-protein coding RNA 00460; siRNA, small interfering RNA.

and $0.67 \pm 0.08$, respectively; $\mathrm{P}<0.05$; Fig. 4A). Similar results were observed using the Transwell migration assay $(\mathrm{P}<0.05$; Fig. 4B and C), which suggested that the genetic knockdown of LINC00460 significantly decreased the migration of OS cells. In addition, the invasive ability of MG-63 and U2-OS cells was significantly decreased by $\sim 61.4$ and $\sim 83.1 \%$, respectively, following transfection with siRNA-LINC00460 compared with their respective siRNA-NCs (Fig. 4B and C). These data indicated that the invasive ability of OS cells transfected with siRNA-LINC00460 was significantly inhibited.

Decreased activity of MMP-9 may affect cell invasion and migration following siRNA-LINC00460 transfection. MMPs are a family of zinc- and calcium-dependent endopeptidases that selectively degrade components of the extracellular matrix (27). MMPs serve crucial roles in tumor cell progression; in particular, MMP-9 is a $92-\mathrm{kDa}$ gelatinase that has been implicated in tumor invasion, growth and distant metastasis (28). Provided that gelatin is an important substrate of MMP-9, a gelatin zymography assay was used to analyze MMP activity to further investigate the effect of LINC00460 on the invasive and migratory capacity of OS cells. Weaker gelatinolytic intensity was observed in MG-63 and U2-OS cells transfected with siRNA-LINC00460 compared with their respective siRNA-NC groups (Fig. 4D). These findings indicated that MMP-9 activity was decreased in the siRNA-transfected cells and thus, that the knockdown of LINC00460 suppressed the OS cell invasive and migratory 
A

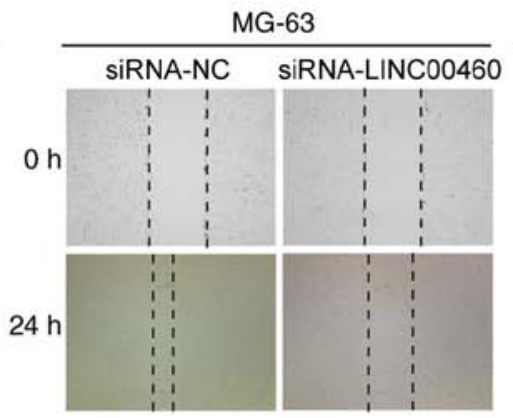

B

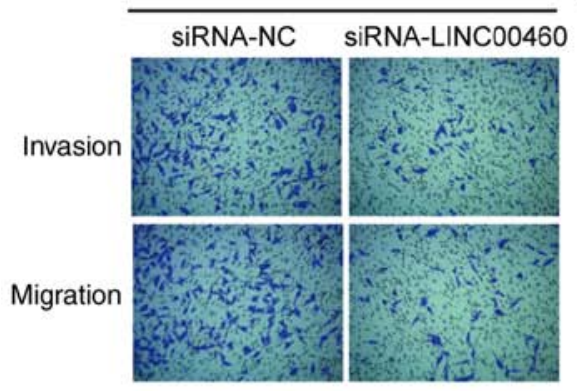

U2-OS

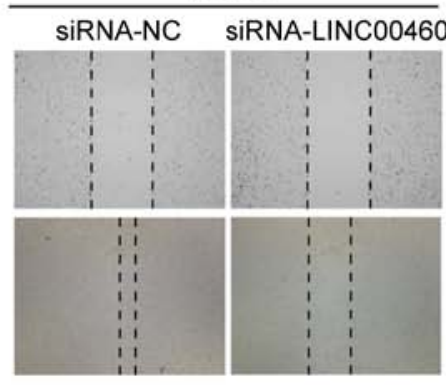

U2-OS

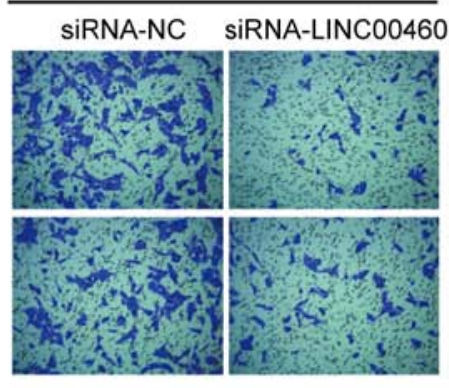

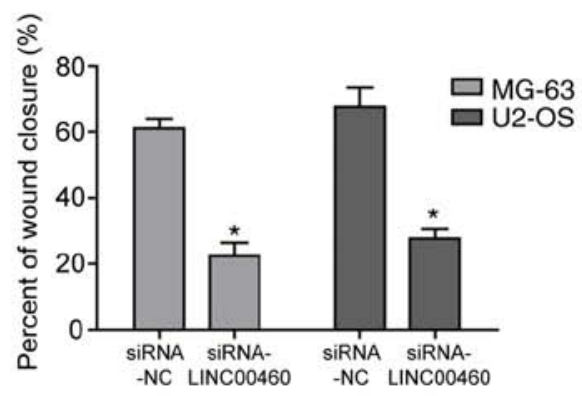
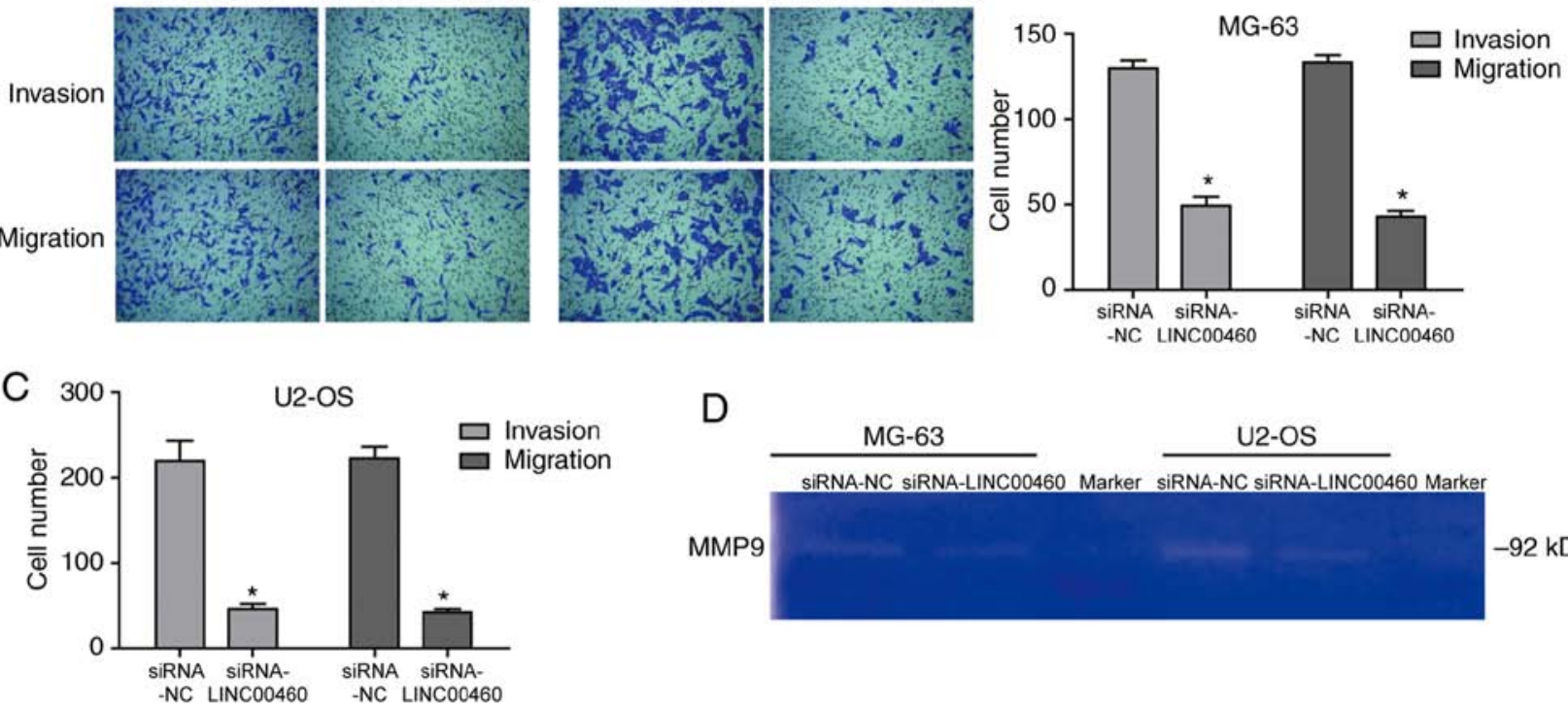

$\mathrm{D}$

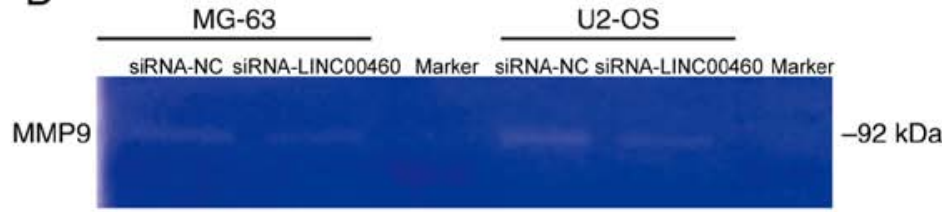

Figure 4. Genetic knockdown of LINC00460 inhibits the migratory and invasive ability of osteosarcoma cells. (A-C) Migratory ability of MG-63 and U2-OS cells transfected with siRNA-NC or siRNA-LINC00460 were evaluated by wound healing and Transwell assays. (A) Representative micrographs (magnification, $\mathrm{x} 40$ ) of the migrated MG-63 cells and U2-OS cells are displayed and the quantification analysis of the percentage of wound healing of migrating MG-63 and U2-OS cells is presented. (B and C) Invasive ability of MG-63 and U2-OS cells transfected with siRNA-NC or siRNA-LINC00460 was measured using Matrigel Transwell assays. Representative micrographs of the the lower chamber of the Transwell plate (magnification, x100) and the quantification analysis of the invasive potential of MG-63 and U2-OS cells are presented. (D) MMP-9 activity in MG-63 and U2-OS cells transfected with siRNA-NC or siRNA-LINC00460 was analyzed by gelatin zymography. All data are presented as the mean \pm SD of three independent experiments. ${ }^{*} \mathrm{P}<0.05 \mathrm{vs}$. NC. NC, negative control; siRNA, small interfering RNA.

ability which may be caused by the decreased activity of MMP-9.

Genetic knockdown of LINC00460 inhibits the EMT process. To further confirm whether the suppression of the EMT process could represent a mechanism behind the siRNA-LINC00460-mediated inhibition of cellular migration and invasion in OS cells, western blot analysis was used to examine the expression of EMT marker proteins. siRNA-LINC00460 transfected MG-63 and U2-OS cells were observed to have significantly increased expression levels of E-cadherin, an epithelial marker, and significantly downregulated expression levels of $\mathrm{N}$-cadherin and vimentin mesenchymal markers compared with their respective siRNA-NC groups (Fig. 5A and B). Furthermore, there was a significant decrease in Slug, a mediator of EMT, in both cell lines transfected with siRNA-LINC00460 compared with siRNA-NC groups $(\mathrm{P}<0.05$; Fig. 5A and $\mathrm{B})$. Taken together, these results suggested that the genetic knockdown of LINC00460 inhibited the migratory and invasive properties of OS cells through suppressing EMT and inhibiting the expression of MMP-9.

\section{Discussion}

To the best of our knowledge, there are few previous studies reporting the roles and respective mechanism of LINC00460. In the present study, a siRNA knockdown strategy was used to silence LINC00460 and investigate the effects of LINC00460 on the biological function of OS cells. The knockdown of LINC00460 inhibited cellular proliferation through inducing apoptosis and cell cycle arrest, effectively reducing the cellular viability of OS cells. The rapid growth of cancer cells is a major factor contributing to malignancy (29), thus it is important to explore the mechanism of tumor cell proliferation to improve cancer treatments. Cellular proliferation is tightly regulated by different cyclins and catalytic CDKs in each cell cycle phase (30,31); for example, cyclin D1 interacts with CDK4 and CDK6 to form a cyclin/CDK complex that acts as an 
A
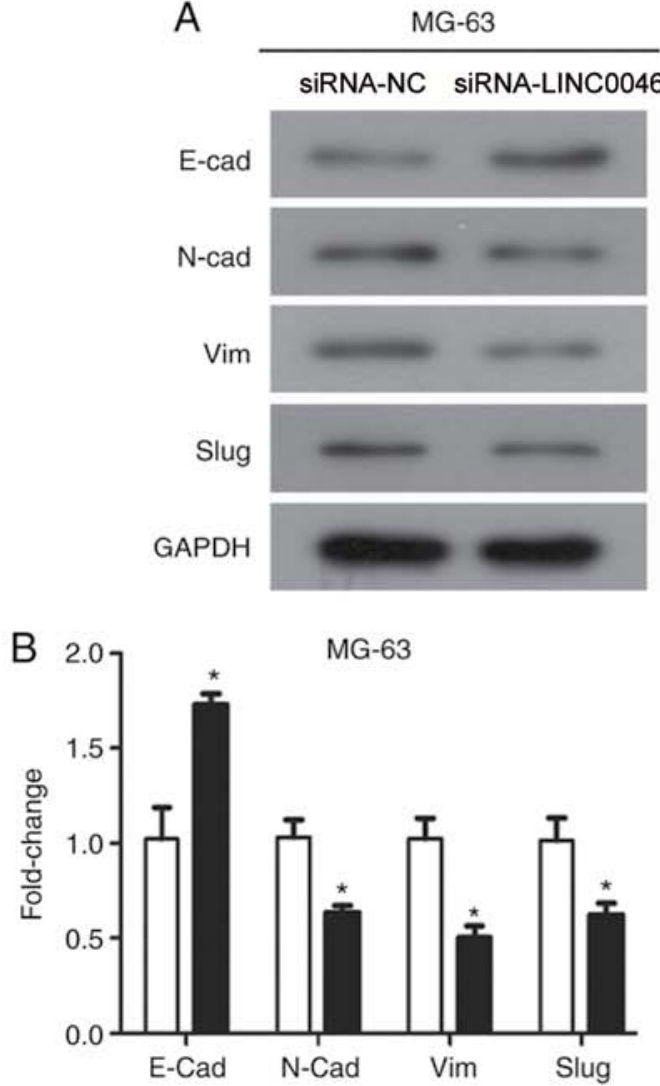

U2-OS
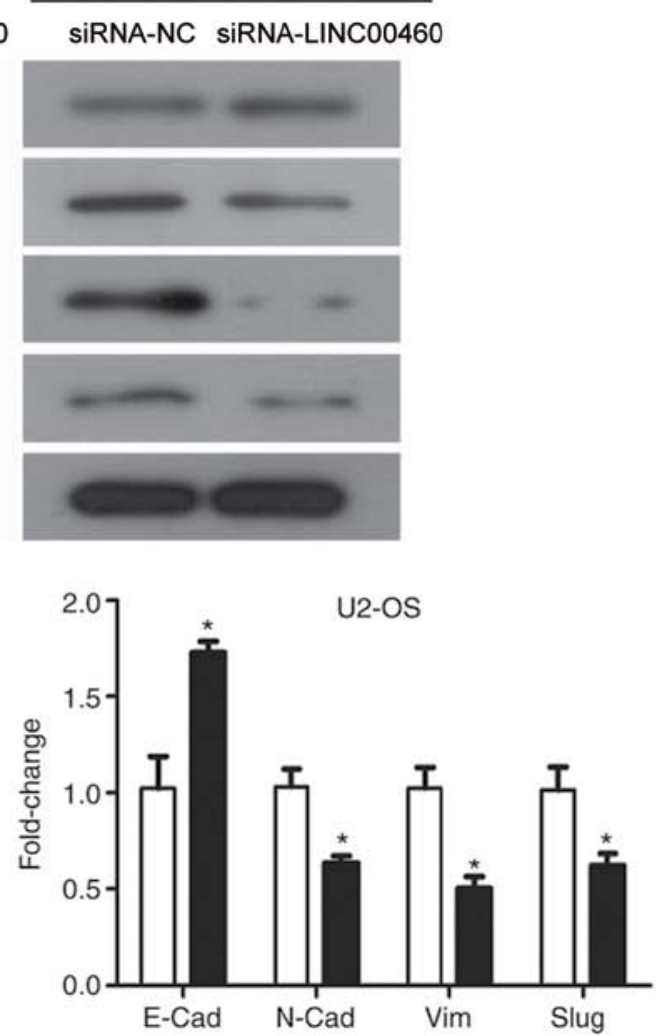

Figure 5. LINC00460 knockdown with siRNA inhibits the EMT process. (A) Western blotting was conducted to analyze EMT marker protein expression levels (E-cadherin, N-cadherin, vimentin and Slug) in MG-63 and U2-OS cells following transfection with siRNA-NC or siRNA-LINC00460. GAPDH was used as the loading control. (B) Semi-quantitative analysis of E-cadherin, N-cadherin, Vimentin and Slug expression levels MG-63 and U2-OS cells following transfection with siRNA-NC or siRNA-LINC00460. Data are presented as the mean \pm SD of three independent experiments. ${ }^{*} \mathrm{P}<0.05$ vs. NC. NC, negative control; EMT, epithelial-mesenchymal transition; siRNA, small interfering RNA.

early regulator to activate downstream gene cascades and drive cells through the G1/S checkpoint (32). Therefore, abnormal levels of cyclin D1, CDK4 and CDK6 are related with a disordered cell cycle. Using flow cytometric analysis, OS cells with silenced LINC00460 gene expression were observed to be arrested in the G0/G1 phase. The underlying mechanisms mediating this were observed to be, at least partly, owing to the reduced expression of cyclin D1 and CDK4/CDK6 in siRNA-LINC00460 transfected cells; thus, LINC00460 is suggested to modulate cell proliferation and/or function in OS partly by upregulating the levels of cyclin D1 and CDK4/CDK6. Kong et al (33) suggested that silencing LINC00460 suppresses nasopharyngeal carcinoma cell proliferation and growth in vitro and in vivo through regulating $\mathrm{miR}-149-5 \mathrm{p} /$ interleukin 6 signaling pathway. Liang et al (19) also reported that silencing LINC00460 suppresses esophageal squamous cell carcinoma cell proliferation and growth through regulating cell cycle and inducing apoptosis, and LINC00460 was regulated by transcriptional co-activator $\mathrm{CBP} / \mathrm{P} 300$ through histone acetylation. The present study results are consistent with the previous research results. However, the underlying mechanisms of carcinogenesis, such as the other molecules involved in this interaction, will require further exploration.

Furthermore, the genetic knockdown of LINC00460 inhibited the migratory and invasive ability of OS cells through reducing MMP-9 expression and inhibiting EMT. MMP-9 is a member of the MMP family, which consists of proteolytic enzymes that selectively degrade all components of the ECM; it is also a biological marker for tumor invasion and metastasis (27). In the present study, MMP-9 activity was observed to be decreased following LINC00460 knockdown with siRNA in OS cells. In addition, EMT, a pivotal biological process in which epithelial cells gradually transform into mesenchymal-like cells through the loss of epithelial markers and the gain of a mesenchyme-like phenotype, serves a crucial role in the induction of cancer cell invasion and metastasis. To investigate whether LINC00460 knockdown decreased the migratory and invasive ability of OS cells through the EMT pathway, the high expression of epithelial markers and the low expression of mesenchymal markers in LINC00460-silenced OS cells was verified. Li et al (20) also demonstrated that LINC00460 promotes cell migration and invasion by inducing EMT in lung cancer cells by physically interacting with heterogeneous nuclear ribonucleoprotein $\mathrm{K}$, whereas it has no effect on cell proliferation. However, this present study has several limitations. Firstly, cultured cells in vitro are unable to simulate the tumor microenvironment in vivo, thus this hypothesis requires further validation using in vivo animal tumorigenesis experiments. Secondly, the present study primarily focused on the action mechanism of LINC00460 knockdown in the progression of OS and additional studies with overexpressed 
LINC00460 gene expression are required to validate these findings.

In conclusion, the present study indicated that LINC00460 functions as an oncogenic factor in OS that may facilitate tumor cell growth, migration and invasion, and inhibit apoptosis. The genetic knockdown of LINC00460 induced cell cycle arrest in the G0/G1 phase, which may in part, be due to its inhibition over the expression of cyclin D1, CDK4 and CDK6. In addition, the knockdown of LINC00460 inhibited the migratory and invasive potential of OS cells, which may be due to the reduced MMP-9 expression and suppressed EMT phenotype observed. These data suggested that LINC00460 upregulation may be a potential risk factor associated with a poor prognosis in OS. However, the downstream target molecules of LINC00460 will require further investigation prior to conclusions being made. Based on these findings, it is proposed that LINC00460 may serve as a potential therapeutic target for OS treatment to obtain an improved prognosis.

\section{Acknowledgements}

Not applicable.

\section{Funding}

No funding was received.

\section{Availability of data and materials}

The datasets used and/or analyzed during the present study are available from the corresponding author upon reasonable request.

\section{Authors' contributions}

JJJ and LPH designed the study; JJJ and FCW performed all the experiments and analyzed the data. LPH wrote the manuscript.

\section{Ethics approval and consent to participate}

Not applicable.

\section{Patient consent for publication}

Not applicable.

\section{Competing interests}

The authors declare that they have no competing interests.

\section{References}

1. Hansen MF, Seton M and Merchant A: Osteosarcoma in Paget's disease of bone. J Bone Miner Res 21 (Suppl 2): S58-S63, 2006.

2. Ottaviani G and Jaffe N: The etiology of osteosarcoma. Cancer Treat Res 152: 15-32, 2009.

3. Mirabello L, Troisi RJ and Savage SA: Osteosarcoma incidence and survival rates from 1973 to 2004: Data from the surveillance, epidemiology, and end results program. Cancer 115: 1531-1543, 2009.
4. Broadhead ML, Clark JC, Myers DE, Dass CR and Choong PF: The molecular pathogenesis of osteosarcoma: A review. Sarcoma 2011: 959248, 2001

5. Zhou G, Shi X, Zhang J, Wu S and Zhao J: MicroRNAs in osteosarcoma: From biological players to clinical contributors, a review. J Int Med Res 41: 1-12, 2013.

6. Costa FF: Non-coding RNAs: Meet thy masters. Bioessays 32: 599-608, 2010

7. He JH, Han ZP and Li YG: Association between long non-coding RNA and human rare diseases (Review). Biomed Rep 2: 19-23, 2014.

8. Chu C, Qu K, Zhong FL, Artandi SE and Chang HY: Genomic maps of long noncoding RNA occupancy reveal principles of RNA-chromatin interactions. Mol Cell 44: 667-678, 2011.

9. Ponting CP and Belgard TG: Transcribed dark matter: meaning or myth? Hum Mol Genet 19: R162-R168, 2010.

10. Rios-Barrera LD, Gutiérrez-Pérez I, Dominguez M and RiesgoEscovar JR: acal is a long non-coding RNA in JNK signaling in epithelial shape changes during drosophila dorsal closure. PLoS Genet 11: e1004927, 2015.

11. Sun J, Lin Y and $\mathrm{Wu}$ J: Long non-coding RNA expression profiling of mouse testis during postnatal development. PLoS One 8: e75750, 2013.

12. Cheetham SW, Gruhl F, Mattick JS and Dinger ME: Long noncoding RNAs and the genetics of cancer. Br J Cancer 108 : 2419-2425, 2013.

13. Gutschner T and Diederichs S: The hallmarks of cancer: A long non-coding RNA point of view. RNA Biol 9: 703-719, 2012.

14. Chan LH, Wang W, Yeung W, Deng Y, Yuan P and Mak KK: Hedgehog signaling induces osteosarcoma development through Yap1 and H19 overexpression. Oncogene 33: 4857-4866, 2014.

15. Sun Y and Qin B: Long noncoding RNA MALAT1 regulates HDAC4-mediated proliferation and apoptosis via decoying of miR-140-5p in osteosarcoma cells. Cancer Med 7: 4584-4597, 2018.

16. Ye JJ, Cheng YL, Deng JJ, Tao WP and Wu L: LncRNA LINC00460 promotes tumor growth of human lung adenocarcinoma by targeting miR-302c-5p/FOXA1 axis. Gene 685: 76-84, 2019.

17. Cao W, Liu JN, Liu Z, Wang X, Han ZG, Ji T, Chen WT and Zou X: A three-lncRNA signature derived from the Atlas of ncRNA in cancer (TANRIC) database predicts the survival of patients with head and neck squamous cell carcinoma. Oral Oncol 65: 94-101, 2017.

18. Zhao G, Fu Y, Su Z and Wu R: How long non-coding RNAs and MicroRNAs mediate the endogenous RNA network of head and neck squamous cell carcinoma: A comprehensive analysis. Cell Physiol Biochem 50: 332-341, 2018.

19. Liang Y, Wu Y, Chen X, Zhang S, Wang K, Guan X, Yang K, Li J and Bai Y: A novel long noncoding RNA linc00460 up-regulated by $\mathrm{CBP} / \mathrm{P} 300$ promotes carcinogenesis in esophageal squamous cell carcinoma. Biosci Rep 37: pii: BSR20171019, 2017.

20. Li K, Sun D, Gou Q, Ke X, Gong Y, Zuo Y, Zhou JK, Guo C, Xia Z, Liu L, et al: Long non-coding RNA linc00460 promotes epithelial-mesenchymal transition and cell migration in lung cancer cells. Cancer Lett 420: 80-90, 2018.

21. Liu X, Wen J, Wang H and Wang Y: Long non-coding RNA LINC00460 promotes epithelial ovarian cancer progression by regulating microRNA-338-3p. Biomed Pharmacother 108: 1022-1028, 2018

22. Livak JK and Schmittgen TD: Analysis of relative gene expression data using real-time quantitative PCR and the 2(-Delta Delta C(T)) method. Methods 25: 402-408, 2001.

23. Lou C, Zhu Z, Zhao Y, Zhu R and Zhao H: Arctigenin, a lignan from Arctium lappa L., inhibits metastasis of human breast cancer cells through the downregulation of MMP-2/-9 and heparanase in MDA-MB-231 cells. Oncol Rep 37: 179-184, 2017.

24. Muthukuru M and Cutler CW: Resistance of MMP9 and TIMP1 to endotoxin tolerance. Pathog Dis 73: pii: ftu003, 2015.

25. Banu SK, Lee J, Starzinski-Powitz A and Arosh JA: Gene expression profiles and functional characterization of human immortalized endometriotic epithelial and stromal cells. Fertil Steril 90: 972-987, 2008.

26. Tang Z, Li C, Kang B, Gao G, Li C and Zhang Z: GEPIA: A web server for cancer and normal gene expression profiling and interactive analyses. Nucleic Acids Res 45 (W1): W98-W102, 2017.

27. Wick W, Platten $M$ and Weller M: Glioma cell invasion: Regulation of metalloproteinase activity by TGF-beta. J Neurooncol 53: 177-185, 2001. 
28. Hua $\mathrm{J}$ and Muschel RJ: Inhibition of matrix metalloproteinase 9 expression by a ribozyme blocks metastasis in a rat sarcoma model system. Cancer Res 56: 5279-5284, 1996.

29. Zhou JJ, Xie Y, Zhao Y and Li ZX: Neuron specific enolase gene silencing suppresses proliferation and promotes apoptosis of lung cancer cells in vitro. Nan Fang Yi Ke Da Xue Xue Bao 31: 1336-1340, 2011 (In Chinese).

30. Hall M and Peters G: Genetic alterations of cyclins, cyclin-dependent kinases, and Cdk inhibitors in human cancer. Adv Cancer Res 68: 67-108, 1996.

31. Sherr CJ: Cancer cell cycles. Science 274: 1672-1677, 1996.
32. Sherr CJ and Roberts JM: CDK inhibitors: Positive and negative regulators of G1-phase progression. Genes Dev 13: 1501-1512,1999.

33. Kong YG, Cui M, Chen SM, Xu Y, Xu Y and Tao ZZ: LncRNA-LINC00460 facilitates nasopharyngeal carcinoma tumorigenesis through sponging miR-149-5p to up-regulate IL6. Gene 639: 77-84, 2018.

c) () $\Theta$ This work is licensed under a Creative Common Attribution-NonCommercial-NoDerivatives 4.0 International (CC BY-NC-ND 4.0) License. 